\title{
Understanding Young Children's Health Beliefs and Diabetes Regimen Adherence
}

\author{
DENISE CHARRON-PROCHOWNIK, PhD; MARSHALL H. BECKER, PhD; \\ MORTON B. BROWN, PhD; WEN-MIIN LIANG, MA; SCARLETT BENNETT
}

Previous studies of chronic illness management in children have focused mainly on parents' health beliefs. However, children's health beliefs also can be an important factor in predicting adherence. Indeed, children 6 to 10 years old spend most waking hours away from home, are under less parental supervision, and are becoming more responsible for their own care. The purpose of this study was to develop a pictorial, multi-item instrument to measure dimensions of the Health Belief Model (HBM) and self-efficacy (SE), designed specifically for children with diabetes, thus making it possible to examine both the parent's and child's health beliefs; to explore the relationship between their beliefs; and to examine the extent to which these beliefs are predictors of adherence and metabolic control.
For children with diabetes who need insulin, the treatment plan is complex, will last a lifetime, and requires numerous behavioral adjustments.' Diabetes potentially can alter lifestyles and well-being. It is not surprising that most studies of patients with diabetes reveal disturbingly low levels of regimen adherence. ${ }^{2}$ Among school-age children and adolescents with diabetes, nonadherence rates range from $40 \%$ to $80 \%$. $^{3}$

People develop a variety of health-related beliefs and attitudes that affect their decisions about the necessity and the desirability of following professional advice. Many of these beliefs are learned from parents and peers and through personal experiences. ${ }^{2}$ Patients' beliefs about the threat of an illness, and about the value and cost of the prescribed regimen, were presumed to be factors in the level of adherence. ${ }^{4}$ Furthermore, evidence of the predictive value of health beliefs for adherence appears strongest when the beliefs of parents, particularly mothers, are examined in relation to a regimen prescribed for children. ${ }^{4}$ This finding not only applies to the mother's direct behavior affecting the regimen, but to the child's own habits and behaviors as well. ${ }^{4}$

The Health Belief Model (HBM), ${ }^{5}$ derived from psychological theories, hypothesized that behavior depends mainly upon the value placed by an individual on a particular goal, and the individual's estimate of the likelihood that a given action will achieve that goal.5. The HBM, originally formulated to explain preventive health actions, later was applied to prescribed therapies and sick-role behaviors. With these later explanations, the nature of events underlying beliefs about health problems was extended to include a diagnosed condition, such as diabetes, which reflected a different type of threat. ${ }^{4}$

In previous applications, the HBM provided a useful framework of psychosocial variables that were predictors of patient adherence, and that may therefore serve as a logical basis for educational interventions. ${ }^{2}$ The model assumes that health is valued, and that cues for action are prevalent. ${ }^{6}$ The four major dimensions (beliefs) of the model, as they apply

From the University of Pittsburgh Westem Psychiatric Institute and Clinic, Pittsburgh, Pennsylvania (Dr Charron-Prochownik), and the University of Michigan School of Public Health and the Michigan Diabetes Research and Training Center, Ann Arbor, Michigan (Drs Becker and Brown, Ms Liang, and Ms Bennett).

This study was supported in part by a grant from the National Institute of Diabetes, Digestive and Kidney Diseases, NIH grant NIH-5P60-DM1-20572.

Correspondence to Denise Charron-Prochownik, PhD, c/o Dr. Kovacs' Office, Western Psychiatric Institute and Clinic, 3811 O'Hara Street, Pittsburgh, PA 15213.

Reprint requests to The Diabetes Educator, 500 North Michigan Avenue, Suite 1400, Chicago, IL 60611-3796. 
to diabetes, are: perceived susceptibility and severity of insulin-dependent diabetes mellitus (IDDM) and of its acute complications (threat), and perceived benefits of and barriers to the treatment management (threat reduction). According to the model, individuals with diabetes will most likely adhere to the treatment plan if they have a motive (eg, are concerned about their health), and if they hold the following four beliefs to be true: they are susceptible to problems due to IDDM; IDDM could have a serious, negative impact on their lives; adherence to professional recommendations will be beneficial in reducing the threat of diabetes-related problems; and the difficulties (barriers) associated with following the health recommendations (eg, cost, pain, side effects) are outweighed by the benefits. In a review by Janz and Becker, ${ }^{6}$ perceived barriers proved to be the most powerful predictor of all HBM dimensions across the various study designs and behaviors. Similar conclusions were drawn by Brownlee-Duffeck and colleagues ${ }^{8}$ in their study with adolescents and diabetes adherence. Kurt, ${ }^{9}$ however, found that severity received the strongest support among the HBM components for predicting overall diabetes adherence.

Although the studies reviewed by Janz and Becker ${ }^{6}$ provided substantial empirical evidence supporting HBM dimensions as important contributors to the explanation and prediction of health behaviors related to a diabetes regimen, this evidence is limited to accounting for as much of the variance in an individual's health-related behaviors as can be explained by these particular attitudes and beliefs. Other variables, not included in the HBM, such as self-efficacy (SE) have been found to fit conceptually within the HBM framework, and to be strong predictors of health behaviors. ${ }^{10}$ Self-efficacy is defined as "the conviction that one can successfully execute the specific behavior required to produce the specific outcome."11 For children with IDDM, self-efficacy can be operationalized as how sure they are of their ability to perform diabetes-related behaviors. According to Bandura, " SE affects the amount of effort a person will expend on a task, and the length of time a person will persist. Thus, the lower the level of SE, the greater the likelihood of not following recommendations, which can be viewed as a barrier. The literature supports the importance of SE in helping to account for the initiation and maintenance of behavioral changes, " such as those associated with diabetes. One previous study ${ }^{12}$ found a positive relationship between SE and diabetes self-care in adults, while a study of teenagers ${ }^{13}$ found a significant positive correlation between SE and metabolic control only for adolescent girls. Rosenstock ${ }^{10}$ concluded that an expanded HBM that incorporates perceived self-efficacy should provide a more powerful approach to understanding and influencing health-related behavior than has been available to date, and that such an exploration should rank high on any research agenda for studying and improving adherence.

Kirsch $t^{4}$ offered additional direction for research relative to the HBM. He argued that because the HBM had not consistently demonstrated predictive validity in relation to adherence to medical regimens, future studies would need a more specific assessment of adherence, incorporating different aspects of the regimen that all should be related to a specific health outcome. Others ${ }^{1+15}$ have argued as well for disease-specific measures rather than for global self-reports or health status measures (eg, glycemic control) as indices of diabetes-related adherence, and the use of standardized measures involving self-reports of specific regimen behaviors (eg, insulin, diet, self-monitoring of blood glucose [SMBG]). In addition, Kurtz ${ }^{9}$ suggested multiple measures of adherence, including an objective indicator (eg, observable behaviors).

Although there have been several investigations in which the HBM has been applied to understanding adherence to a diabetes regimen in adults ${ }^{1,16-18}$ and in adolescents, ${ }^{19,20}$ very few studies have focused on children. In a study with parents, Ludvigsson et $\mathrm{a}^{21}$ found that the threat-reduction component of the HBM influenced adherence behaviors in children with diabetes. It does not appear that this study (or any other) explored both the parent's and the child's health beliefs simultaneously. Furthermore, most of the research in the area of parent's health beliefs and adherence with childhood chronic illness has focused on diseases other than diabetes, such as asthma ${ }^{22.23}$ and orthodontics. ${ }^{24}$ In addition, none of these studies have included self-efficacy in their conceptual frameworks.

Research directed at understanding adherence in children with diabetes must include the child's own perceptions of health beliefs and behaviors, because the school-age child 6 to 11 years old (schooler) spends most waking hours away from home in school, recreational activities, sports, or with friends. These children no longer are under the continuous supervision of their parents, and are becoming self-caring and more responsible. The schooler is in Piaget's Concrete Operational Stage. ${ }^{25}$ Ginsburg ${ }^{25}$ describes children in this stage as: developing an understanding of relationships between events, things, and objects, and understanding cause and effect; applying rules/regulations, and distinguishing right from wrong; discussing problems that they face, questions about life, and attitudes. The schooler also has a very basic, concrete understanding of the body and its functions. Several of the diabetes self-management skills are being acquired during this developmental period. The age ranges associated with the mastery of diabetes skills as recommended by the American Diabetes Association ${ }^{26}$ are: recognizes/reports hypoglycemia, age 8 to 10 years; treats hypoglycemia, age 10 to 12 years; performs blood glucose testing, age 8 to 10 years; draws dose with one insulin type, age 10 to 12 years; and identifies appropriate snacks, age 10 to 12 years. In a study by Wysocki and colleagues, ${ }^{27} 25 \%$ to $50 \%$ of 5-to-7-year-old children were found to assist with SMBG; know insulin schedules; recognize report, and treat low blood sugars; state the role of diet and insulin; assist with insulin administration; and categorize foods into groups.

Because of increasing maturity, independence, and responsibility for their own actions, the schooler's perceptions of health beliefs can become an important factor in predicting adherence to the diabetes regimen. Gochman ${ }^{28.29}$ has conducted studies exploring the perceptions of the HBM with well children, ages 6 to 11 years. He and others ${ }^{30-32}$ who conduct research with children have used pictorial instruments, and have found that children interact very well with this format.

Our intent was to address some of the issues raised by previous researchers in this field. We designed a study that directly examines children's health beliefs, strengthens the 
HBM by incorporating self-efficacy as a predictor variable, and assesses specific regimen behaviors related to diabetes adherence. One objective of this study was to develop multiitem instruments to measure dimensions of the HBM and SE designed specifically for children with diabetes. These measures allowed us to examine both the child's and parent's beliefs regarding diabetes, to explore the relationship between a child's and parent's health beliefs, and to examine the extent to which these beliefs are predictors of diabetes adherence and metabolic control. An additional goal was to examine relationships among the outcome variables: adherence, metabolic control, and well-being.

\section{Methods}

Sample and Procedure The subjects for the study were 50 pairs, each consisting of a child and the child's parent. Potential candidates were children between the ages of 6 and 9 years who had IDDM for at least 6 months, with no significant additional chronic illness. Subjects were recruited from the patient population at a pediatric diabetes clinic of a major teaching hospital in the Northwest. One week prior to their scheduled clinic appointment, potential candidates were telephoned by the project director, who explained the study and the role of participants. The refusal rate was $3 \%$. Informed written consent was obtained during clinic visits from both parent and child.

The sample was composed of 28 boys and 22 girls, with a mean age of 7.8 years $(S D=1.1)$. The mean duration of IDDM was 3.4 years ( $S D=1.8$, range $=1$ to 8 years). Their glycosylated hemoglobin levels ranged from $6.9 \%$ to $17 \%$ (mean $=11.4 \%, \mathrm{SD}=2.3)$. Most children were white $(92 \%)$, from families with two parents present in the home (72\%), with an average of 1.6 siblings $(\mathrm{SD}=1.2$, range $=0$ to 5 ).

Data were collected separately from each child and parent immediately following a routine visit at the pediatric diabetes clinic. The child's interview was conducted by the project director in a private room in the diabetes clinic. This interview lasted approximately 15 minutes. Upon completion, the child was given a printed certificate thanking him/her for participating in the study. During the child's interview, the parent (in an adjacent room) completed a self-administered questionnaire that required approximately 20 minutes to respond. Once the interview and questionnaire were completed, the child proceeded to the blood-drawing station where a routine laboratory blood test (eg, glycosylated hemoglobin) was obtained.

Evaluation Measures Multidimensional, multi-item instruments were developed to assess dimensions of the HBM and SE, designed specifically for children with diabetes and their parents. In addition, separate measures of adherence, metabolic control, and well-being were obtained.

Childrens Health Beliefs The instrument, The Diabetes Health Belief Pictorial Instrument for School-Age Children (HB Picture Scale), was developed for this study to measure school-age (approximately 5 to 10 years old) children's diabetes-related health beliefs (severity, susceptibility, benefits, barriers, and self-efficacy). The pictorial instrument has a format similar to that of Biggs and Garrison' $\mathrm{s}^{31}$ Diabetes and Me Knowledge/Attitude/Behavior Scale, and to Harter's ${ }^{30}$
Perceived Competence and Social Acceptance Scales. Both of these instruments were described by their authors as direct and developmentally-sensitive measures for children. Younger children can better comprehend ideas represented in the familiar medium of pictures and stories..$^{30,31,33}$ Cartoons are visual, concrete, and have been shown to be an effective data collection method with this age group, ${ }^{30-33}$ stimulating interaction between the child and interviewer about diabetes. ${ }^{31}$

The HB Picture Scale is forced-choice and bipolar, with four-level response categories. The child is shown 11 pairs of pictures, each displaying opposing behaviors (Figure 1). The pictures in the booklet are facing the child while the text faces the interviewer. Similar to the procedure described by Biggs and Garrison, ${ }^{31}$ the interviewer begins by saying, "This is something like a picture game." The participants are told that the children in these pictures have diabetes. The interviewer explains what the child in each picture frame (Figure 1) is doing. For example, the interviewer points to the left frame and says, "This child has enough time to take all his/her insulin shots," then points to the right frame and says, "This child is too busy to take all his/her insulin shots. Which one is more like you?" The child projects the character's behaviors onto their beliefs about themselves. The child is instructed to select one of the two frames, and is thus forced to choose between pictures depicting two opposing anchors (bipolar).

A four-point response scale is used that coincides with the pictures (Figure 1). Two responses are at opposing ends (eg, Always versus Hardly Ever), with the two other selections in between, representing lesser magnitudes of an anchor. For example, the four responses for this item are: Always Too Busy, Usually Too Busy, Sometimes Too Busy, or Hardly Ever Too Busy. Thus, after the child points to one picture, the interviewer directs the child to point to a circle directly below that picture (in order to obtain the more specific response). The number of the chosen circle is recorded for the corresponding item on the Children's Health Belief Scoring Sheet (Figure 2).

The HB Picture Scale has a total of 11 items, with 2 items for each of the 4 health-belief dimensions, and 3 items reflecting self-efficacy. A total of five subscale scores were generated from these responses. Each HBM subscale had a total score ranging from 2 to 8, with lower scores representing stronger beliefs. Self-efficacy scores ranged from 3 to 12 , with higher scores reflecting stronger self-efficacy. The direction of magnitude of the response scale mirrors that of the parent's instrument.

Parent and child had parallel items; the child's instrument had simplified wording with illustrated cartoons. Unlike the child's instrument with two items per dimension, parents had four items per dimension.

Parents'Health Beliefs Parents' health beliefs about their child's diabetes were measured by The Diabetes and Health Belief Instrument, modified for this study for parents from the Becker and Janz 16-item Health Belief Model Scale ${ }^{2}$ after the Given et al ${ }^{18} \mathrm{HBM} 76$-item Scale. The Diabetes and Health Belief Instrument is a close-ended instrument consisting of 21 items. Each item is scored on a Likert scale, ranging from 1 (Strongly Agree) to 5 (Strongly Disagree). 


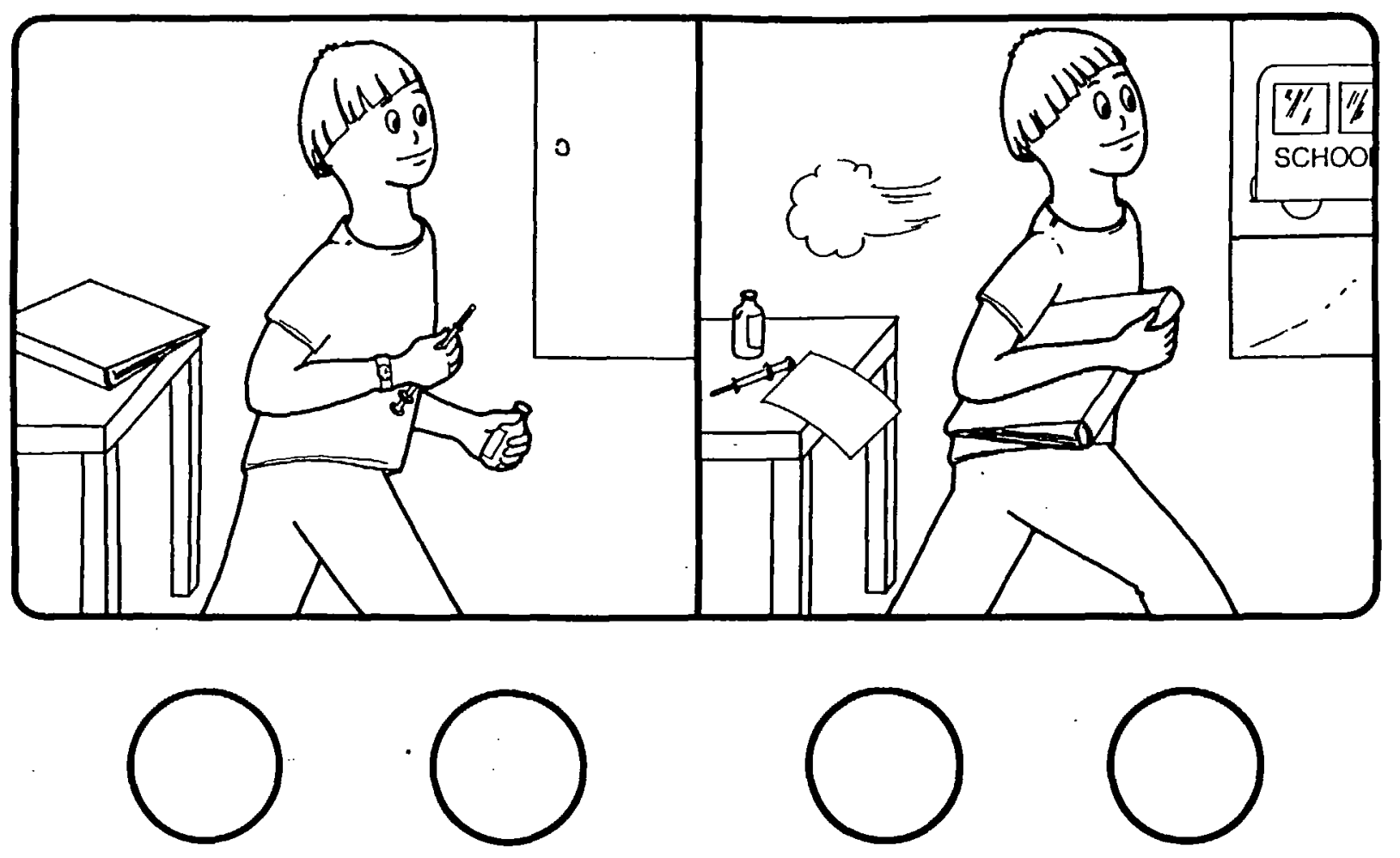

This child is too busy to take all his/her insulin shots

This child has enough time to take all his/her insulin shots

When it comes to taking your insulin shots, are you:

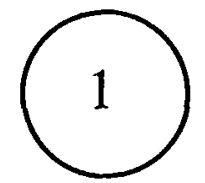

always too busy

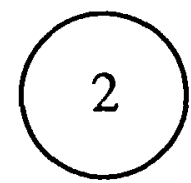

usually too busy

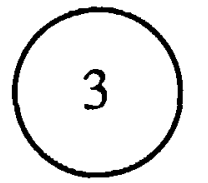

sometimes too busy

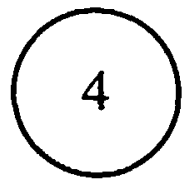

hardly ever too busy

Fig 1. Example of a "barrier item" from the Diabetes Health Belief Pictorial Instrument for school-age children. 
ID

Name

Date

\section{Children's Health Belief Scoring Form}

\section{ITEM DESCRIPTION}

1 Always take care (sev)

2 Always sick (sev)

3 Blood sugars too high (susc)

4 Chance of reactions (susc)

5 Too busy (barr)

6 Doesn't know food plan (barr)

7 Insulin: Blood sugars just right (ben)

8 Diet: Blood sugars just right (ben)

Severity Score:

Susceptibility Score:

Barrier Score:

Benefit Score:

9 Sure to take snacks (eff)

10 Sure to help with shots (eff)

11 Sure to help with blood sugars (eff)

Self-Efficacy Total Score:
RESPONSE

(circle one)

$\begin{array}{ll}3 & 4 \\ 3 & 4 \\ 3 & 4 \\ 3 & 4 \\ 3 & 4 \\ 3 & 4 \\ 3 & 4 \\ 3 & 4\end{array}$

Additional Notes:

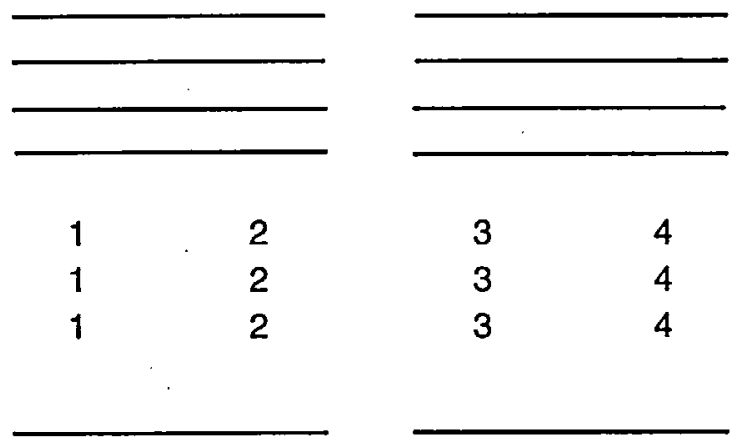

4

4

4

4

4

4

4

Fig 2. The scoring form for the Diabetes Health Belief Pictorial Instrument for school-age children.

Each health belief dimension is an individual subscale derived from a summary score based on four items; lower scores reflect stronger beliefs. The Given et al Scale ${ }^{18}$ has been shown to be reliable and valid. An example of a typical item is, "Taking insulin shots interferes with my child's normal daily activities."

Parent's self-efficacy was measured by The Parent's SelfEfficacy in Diabetes Management Scale. This four-item, close-ended measure was modified for this study from the Diabetes and Pregnancy Questionnaire. ${ }^{34}$ A sample item is, "How confident are you that you'll be able to give all the recommended insulin shots?" Item scores were obtained from a bipolar scale ranging from 10 (Very Confident) to 0 (Not At All Confident). Süms of the items ranged from 0 to 40 , with 40 indicating the highest level of confidence (selfefficacy) in performing diabetes-related tasks.

Adherence Adherence to the diabetes treatment regimen was operationally defined as the extent to which self-care behavior (performed by child and/or parent) for diabetes management coincides with the diabetes regimen recommended by the health care team (modified from Haynes, Taylor, and Sackett ${ }^{35}$ ) to achieve metabolic control. For example, some questions that reflected adherence were, "What were you told to do? What did you do?" Adherence was measured by both subjective (self-report) and objective (observable or physiological) indicators. Subjective data were obtained from parent and child during separate interviews. The Diabetes Management Questionnaire, developed for this study, focused on the dimensions of frequency and timing over the past week (before the clinic visit) of the major components of the diabetes regimen (insulin administration, meal plan, and self-monitoring of blood glucose), as recommended by the health care professional. In previous studies ${ }^{15,36,37}$ that measured diabetes regimen adherence with 6 to 19 year olds, the younger age group were found to be reliable reporters about behaviors that require qualitative recall and frequency recall, but not as reliable about timing. A composite score from the individual components was created; lower scores indicated greater adherence. 
Table 1. Correlations Among Child and Parent Health Beliefs, Adherence, and Metabolic Control

$\begin{array}{lccc}\text { Child Health Beliefs } & \text { Reported Adherence } & \text { Objective Adherence } & \text { GHb } \\ \text { Susceptibility } & .16 & .07 & .05 \\ \text { Severity } & -.08 & -.01 & .31^{*} \\ \text { Benefit } & -.04 & -.05 & .09 \\ \text { Barrier } & -.13 & .31^{*} & -.05 \\ \text { Self-Efficacy } & .01 & .36^{*} & .01 \\ \text { Parent Health Beliefs } & & & \\ \text { Susceptibility } & .12 & -.28 & .24 \\ \text { Severity } & -.06 & -.06 & .02 \\ \text { Benefit } & .00 & -.49^{*} & .27 \\ \text { Barrier } & .07 & .47^{*} & -.19 \\ \text { Self-Efficacy } & -.14 & .55^{*} & -.22\end{array}$

${ }^{*} P \leq .05$

The first objective indicator measured observable behaviors: child wears medic alert bracelet, brought record of blood sugar readings (log or memory meter), carries sugar source in case of reaction, and an actual count of the blood sugars done over the past week. The scores for these items were added together; higher scores indicated greater adherence. The second objective indicator was an indirect, objective measure: metabolic control, assessed by the physiological indicator of total glycosylated hemoglobin assays $(\mathrm{GHb})$, determined by boronate affinity chromatography (Glyc-Affin GHb, Isolab, Inc, Akron, $\mathrm{OH}$ ). The normal range for this assay in our laboratory was $5.0 \%$ to $8.0 \%$.

Children's responsibility for their own self-care management was assessed by three items from the general information form. Parents were asked, "Does your child take responsibility for giving him/herself insulin? doing his/her own blood sugar testing? remembering snacks?" Responses were scored on a scale of 1 (Almost All the Time), 2 (Some of the Time), and 3 (Never). Responsibility scores ranged from 3 to 9, with lower scores representing greater responsibility. For content consistency, these questions were similar to the three items from the child's self-efficacy subscale, and to the three items from the adherence measure.

Well-Being Well-being, a measure of quality of life, was defined as a perception of one's general health. This self-rating was assessed by a nine-step ladder scale (modified from Andrews and Withey ${ }^{38}$ ), ranging from a top step of 9 (Healthy and Strong), to 5 (Not Too Strong, But Not Too Weak), to a bottom step of 1 (Sick and Weak). These three steps also were depicted by small silhouettes of people, in which number 9 had a standing person, number 5 had a sitting person, and number 1 had a person lying down. The children were instructed by the interviewer to point to the step that shows "how well you feel."

Parents also were asked to rate their perception of their child's general health using a similar ladder scale. Like the child's, it ranged from a top step of 9 (Best Health My Child Would Expect to Have) to a bottom step of 1 (Worst Health My Child Would Expect ot Have). For both child and parent scales, higher scores indicated better health.
Analyses All items were precoded as multiple-choice. Data from each form were double entered. The data files from the individual forms were merged to create a single data file. The usual Pearson correlation coefficient was used to assess association. All possible subset regression was used to identify potential multiple linear regression models within a specified subset of the variables. The models of interest then were fitted by ordinary multiple linear regression. Cronbach's alpha was used to measure internal consistency within a scale. All calculations were performed by either BMDP or.SPSS statistical software.

\section{Results}

Psychometric Properties of the Health Belief Instrument Content validity estimates for the HB Picture Scale were determined by a panel of four experts who specialized in the HBM and/or childhood diabetes. They reviewed the instrument several times during its development phase for both pictorial and text accuracy. Changes were made according to their recommendations.

Distribution of item scores were determined by the response score ranges and the frequency distributions. Several variables had a very concentrated distribution with primarily only one value being used, making it difficult to estimate accurate correlations. Most of these distributions were positively skewed, indicating more socially acceptable responses. Therefore, inter-item associations and discriminating validity were not assessed.

Criterion-related validity (concurrent) was determined by the intercorrelation of the HBM subscales (dimensions) with adherence and metabolic control. The correlations among these variables are presented in Table 1 .

The Parent's Diabetes and Health Belief Instrument was modified carefully from the Becker and Janz Health Belief Scale $^{2}$ to maintain its integrity. Similar caution was taken when modifying The Parent's Self-Efficacy Scale from The Pregnancy and Diabetes Questionnaire. ${ }^{34}$ Both modified scales were evaluated by a panel of experts for representativeness of content and face validity. Cronbach's alpha coefficients for the health belief subscales (dimensions) ranged from .24 to .75 . The alpha coefficient for the 
Table 2. Descriptive Analysis of Health Belief Subscales

\begin{tabular}{lccc} 
Children's HB Subscales & Mean (SD) & Possible Item Range & Selected Item Range \\
Susceptibility & $2.9(.44)$ & $1-4^{*}$ & $2.0-3.5$ \\
Severity & $2.2(.51)$ & $1-4^{*}$ & $1.0-3.5$ \\
Benefit & $1.8(.53)$ & $1-4^{*}$ & $1.0-3.5$ \\
Barrier & $3.5(.38)$ & $1-4^{*}$ & $2.5-4.0$ \\
Self-Efficacy & $3.5(.41)$ & $1-4 \dagger$ & $2.7-4.0$ \\
Parents'HB Subscales & & & $1.0-2.0$ \\
Susceptibility & $1.4(.29)$ & $1-5 \ddagger$ & $1.3-3.5$ \\
Severity & $2.3(.45)$ & $1-5 \ddagger$ & $1.0-2.0$ \\
Benefit & $1.3(.32)$ & $1-5 \ddagger$ & $2.0-5.0$ \\
Barrier & $4.1(.66)$ & $1-5 \ddagger$ & $4.0-10$ \\
Self-Efficacy & $9.0(1.2)$ & $0-10 \S$ &. \\
Subscale scores determined by the mean of the iterns in subscale. & & & \\
*1-4 (lower score $=$ stronger belief) & & & \\
$\dagger 1-4$ (higher score $=$ higher self-efficacy) & & & \\
$\ddagger 1-5$ (lower score $=$ stronger belief) & & & \\
\$1-10 (higher score $=$ higher self-efficacy) & & & \\
\hline
\end{tabular}

sèlf-efficacy scale was .69. Except for susceptibility and severity subscales, for which the alphas were below .50 , alpha coefficients had a moderate level of internal consistency and marginally acceptable levels for subscales and scales in early stages of research. ${ }^{39}$

Descriptive Analyses Overall, children tended to report moderate to very strong health beliefs about susceptibility to and severity of diabetes and its acute complications, about benefits of the treatment regimen, and high levels of self-efficacy. A summary of these results, as indicated by means and SDs, is found in Table 2.

Parents tended to have stronger overall health beliefs than those of the children and also reported high levels of self-efficacy in performing diabetes-related management tasks. Both children and parents tended to report experiencing few barriers. Similarly, children and parents reported high levels of adherence, but the overall sample mean for the observable objective measures of adherence was only moderate.

The results indicate that $44 \%$ of the children in this study take responsibility at least some of the time for their insulin injections, $78 \%$ for blood sugar monitoring, and $92 \%$ for remembering snacks. These children have an average of 17 blood sugars done per week (range 0 to 30 ). Seventy-six percent of the children rated their overall health greater than 7 on a scale of 1 to 9 (in which 1 is worst), while only $44 \%$ of the parents rated their child's health greater than 7. Parents rated their child's diabetes control as follows: $50 \%$ very good to excellent, $28 \%$ good, and $22 \%$ fair.

Bivariate Analyses Pearson correlation coefficients ( $r$ ) were used to test the hypothesized associations between child and parent health beliefs, health beliefs with adherence and metabolic control, and adherence with metabolic control and well-being. Correlations are presented in Table 1.

Results indicate that children's health beliefs were not significantly associated with those of their parents (Pearson $r$ ranged from -.04 to .19 , not shown in Table 1 ). However, children's self-reported adherence was significantly correlated with parents' own perceptions of adherence $(r=.60$, $P<.001)$.

Overall, children's health beliefs were found to have little significant correlation with the outcome variables. Only moderate correlations were noted among some children's health belief dimensions and metabolic control ( $\mathrm{GHb}$ ) and observable adherence. Perceived severity was significantly associated with $\mathrm{GHb}(r=.31, P<.05)$, indicating that a higher belief in severity was related to better metabolic control. Perceived barriers was found to correlate significantly with observed adherence $(r=31, P<.05)$, indicating that greater perceived barriers to treatment were related to greater nonadherence. Self-efficacy also was correlated significantly with observed adherence $(r=.36, P<.02)$, indicating that greater self-efficacy was associated with greater adherence.

Parents' health beliefs had some similar correlational patterns to the health beliefs of the children. Although none of the parents' health belief subscales was significantly associated with $\mathrm{GHb}$, observed adherence was significantly correlated $(P<.001)$ with perceived benefits $(r=-.49)$, perceived barriers ( $r=.47)$, and self-efficacy $(r=.55)$. Parental reporting of greater adherence was associated with stronger belief in the benefits of the treatment plan, fewer barriers, and greater self-efficacy. In addition, parents who rated their children as having better health (higher levels of well-being) had stronger beliefs about susceptibility to acute complications $(r=-.37, P<.01)$ and about benefits of treatment $(r=.49$, $P<.01$ ).

There were no significant associations detected among any of the health belief dimensions with self-reported adherence; therefore, it was not used as a dependent variable in a multiple regression analysis. Well-being was not strongly associated with either adherence or metabolic control. Similarly, child responsibility was not correlated with either $\mathrm{GHb}$ or adherence. Greater child responsibility was significantly related to having more siblings $(r=-.40, P<.01)$ and to being older $(r=-.52, P<.001)$. 
Multivariate Analyses Separate least-squares, multiple regression analyses (MRA) were performed to determine statistically significant predictors of observable adherence and metabolic control from the child and parent health-belief subscales, diabetes-related factors (care responsibility), and demographic variables (SES, family structure, gender, and age). Separate MRA were performed for the two dependent variables (observable adherence and $\mathrm{GHb}$ ) and for the two subsamples (children and parents). Although, a "best" subset model of child health beliefs and parent health beliefs was selected for each outcome variable, alternative models were available.

The best predictive model of observable adherence with children's health beliefs had two predictors. Greater adherence was associated with greater child self-efficacy and higher SES (higher-educated parent[s]). The variables explained $21 \%$ of the variance in adherence, with a multiple $R$ of .46 . None of the other child health beliefs was significantly associated with adherence.

The best predictive model of metabolic control with children's health beliefs also had two variables. Better metabolic control was predicted by stronger beliefs in severity and by higher SES. The variable explained $18 \%$ of the variance in metabolic control $(R=.43)$.

The best predictive model of obșervable adherence with parents' health beliefs had three variables. Greater adherence was associated with lower parental beliefs about barriers, stronger parental beliefs in benefit, and better self-reported adherence. These variables explained $43 \%$ of the variance in adherence $(R=.66)$.

The best predictive model of metabolic control with parents' health beliefs had three variables. Better control was predicted by stronger parental beliefs in benefit, lower child's adherence (as reported by parents), and higher SES. These variables explained $20 \%$ of the variance in control $(R=.45)$.

\section{Discussion}

The HB Picture Scale was easy to administer, and was readily comprehended by children as young as 5 years old. The HB Picture Scale appears to have sufficient face, content, and concurrent validity. However, due to several variables having uneven distributions, it was difficult to accurately assess further psychometric properties. Currently, there are two items representing each health belief dimension. The researchers have decided to add a third item to each subscale to enhance the representativeness of the construct, and to potentiate the variance of each subscale. In addition, a larger sample with a more diverse response pattern is needed. Psychometric analyses also will be repeated in the future with these modifications. At that time, the HB Picture Scale hopefully will have sufficient validity and reliability for use in assessing the health beliefs and predicting the adherence behavior of children with diabetes.

Because the elements of the HBM and self-efficacy are alterable, health care professionals could tailor interventions to suit the particular needs of each patient based on their responses. The instrument also could be used to evaluate the effectiveness of various interventions to alter those health beliefs that influence adherence. In addition to the methodological implications, health beliefs and self-reported adher- ence were assessed directly from young children and their parents. Overall, as predicted by the HBM, both the children and parents reported moderate to very strong health beliefs (eg, diabetes is severe, child is susceptible to problems related to diabetes, and treatment is beneficial), while barriers to management were perceived as low to moderate. Also, both children and parents reported high levels of self-efficacy. Most children felt very confident about being able to assist in performing some diabetes self-care skills. Similarly, parents felt very confident in carrying out the diabetes treatment plan for their children. However, despite the similarities of child and parent health beliefs, they were not significantly correlated.

According to the HBM, individuals who hold the pattern of beliefs described above are most likely to be adherent. As the model hypothesized, this sample of children and parents reported overall high levels of adherence. These results are unlike previous studies ${ }^{3}$ that reported nonadherence rates with diabetes management of $40 \%$ to $80 \%$. Interestingly, the two objective measures for adherence yielded similar results, but were not congruent with self-reported adherence. Observable adherence had an overall mean score in the moderate range, and glycosylated hemoglobin produced a mean of $11.4 \%$, indicating only fair metabolic control. However, both children and parents reported high levels of adherence. This discrepancy can be explained partially by the subjects' need to provide socially acceptable responses. In addition, self-reported adherence was not correlated with any of the health beliefs or other health outcomes. Therefore, objective measures appear to be more valid indicators of adherence. This finding supports the argument for using multiple measures of adherence, especially objective indicators.

Adherence was not significantly associated with the child's management responsibility. Ninety-two percent of the sample had responsibility for at least one of the diabetes tasks some of the time. As expected, children who were older and who had more siblings had greater responsibility.

In our study, children's perception of severity of illness, barriers to treatment, and self-efficacy were significantly correlated with observable adherence or metabolic control, similar to the results reported by Brownlee-Duffeck et al. ${ }^{8}$ Children who believed their diabetes was severe had better metabolic control, similar to Kurtz ${ }^{9}$ who had previously noted severity as the strongest predictor of adherence. As anticipated, children who reported greater barriers to treatment, and who were less confident about performing some diabetes tasks, were more nonadherent.

Parents believing in the benefits of treatment, perceiving fewer barriers to treatment, and having greater confidence in performing tasks were more adherent. Barriers has been found to berthe most powerful predictor of adherence across various studies. ${ }^{2.8}$ In this research, barriers was significantly correlated with adherence for both children and parents. As predicted, self-efficacy also was related to adherence for both children and parents.

According to Revicki, ${ }^{40}$ the control of diabetes and improvement in quality of life (well-being) requires close adherence to a treatment regimen. Surprisingly, results from the present research did not support this assumed correlation. Well-being was not significantly associated with either adherence or metabolic control. 
In the final predictive models, some health beliefs that were significantly correlated with observable adherence and metabolic control did not enter the model due to the presence of other variables. Results from the multiple regression analyses showed that the more compliant children had greater SE and higher-educated parents. This result is similar to that of Hurley and Shea, ${ }^{12}$ who reported SE as a strong predictor of self-care. Children with better metabolic control held stronger beliefs in severity of the condition and had better-educated parents.

The parents' regression models for adherence and control had different predictors. Parents of children who were more adherent (based on observations) saw fewer barriers related to the regimen, had stronger beliefs in the benefit of treatment, and reported greater adherence. Parents of children with better metabolic control also held stronger benefit beliefs and were better-educated, but reported having children that were less adherent.

All of the above predictors were associated with their respective outcome variables in the hypothesized direction, except for better metabolic control which was associated with less-adherent behavior. This result is true only for reported adherence, which showed little association with the objective measures of compliance. Perhaps parents of children with better metabolic control have higher expectations in managing diabetes, and thus have more stringent criteria for measuring adherence.

The parents' adherence model explained a greater percent of the variance (43\%) than did the children's adherence model (21\%). In addition, SES (parental education) was the most frequent predictor across all models.

Due to the small, homogeneous sample used in this study, and the unequal distribution of the children's health belief data, caution should be taken when interpreting and generalizing these results. The findings suggest that the children's models had different health belief predictors from those of the parents. These differences may influence our approaches to teaching and management, supporting the need for separate assessments of children and parents, and to target individual interventions based on their differences. For example, in families that are nonadherent with the diabetes regimen, interventions to enhance $\mathrm{SE}$, such as promoting confidence, can be helpful for children. Obtaining from the parents an assessment of perceived barriers can aid in developing strategies to alleviate the barriers.

We thank Linda Alvira for the illustrations, Denise Garden and Margie Fox for their clinical assistance and support, Jill Peters for technical assistance, Marti Funnell and Dr. Nancy Janz for their suggestions, the entire University of Michigan Pediatric Endocrine Staff, and Dr. Maria Kovacs for providing time and guidance.

\section{References}

1. Cerkoney K, Hart L. The relationship between the health belief model and compliance of persons with diabetes. Diabetes Care 1980;3:594-98.

2. Becker M, Janz N. The health belief model applied to understanding diabetes regimen compliance. Diabetes Educ 1985;11:41-47.

3. LaGreca A, Follensbee D, Skyler J. Developmental and behavioral aspects of diabetes management in youngsters. Children's Health Care 1990;19:132-39.
4. Kirscht J. The health belief model and predictions of health actions. In: Gochman D, ed. Health behavior: emerging research perspectives. New York: Plenum Press, 1988:27-41.

5. Maiman L, Becker M. The health belief model: origins and correlates in psychological theory. In: Becker $\mathrm{MH}$, ed. The health belief model and personal health behaviors. Thorofare, NJ: Charles B. Slack Inc, 1974:9-26.

6. Janz $N$, Becker $M$. The health belief model: a decade later. Health Educ Q 1984;11:26-45.

7. Rosenstock I. Understanding and enhancing patient compliance with diabetes regimens. Diabetes Care 1985;8:610-16.

8. Brownlee-Duffeck $M$, et al. The role of health beliefs in the regimen adherence and metabolic control of adolescents and adults with diabetes mellitus. J Consult Clin Psychol 1987;55:139-44

9. Kurtz S. Adherence to diabetes regimen: empirical status and clinical applications. Diabetes Educ 1990;16:50-56.

10. Rosenstock I, Stretcher V, Becker M. Social leaming theory and the health belief model. Health Educ Q 1988;15:175-83

11. Bandura A. Social leaming theory. New Jersey: Prentice-Hall Inc, 1977.

12. Hurley C, Shea C. Self-efficacy: strategies for enhancing diabetes self-care. Diabetes Educ 1992;18:146-50.

13. Grossman H, Brink S, Hauser S. Self-efficacy in adolescent girls and boys with IDDM. Diabetes Care 1987;10:324-29.

14. Glasgow R. Compliance to diabetes regimens: conceptualization complexity, and determinants. In: Cramer J, Spilker B, eds. Patient compliance in medical practice and clinical trials. New York: Raven Press, 1991:209-24.

15. Johnson S. Methodological considerations in pediatric behavioral research: measurement. Dev Behav Pediatr 1991;12:361-69.

16. Polly R. Diabetes health beliefs, self-care behaviors, and glycemic control among older adults with NIDDM. Diabetes Educ 1992;18:32 I-27.

17. Harris R, Linn M, Sandfer R, Skyler J. Development of the diabetes health belief scale. Diabetes Educ 1987;13:292-97

18. Given C, Given B, Gallin R, Condon J. Development of scales to measure beliefs of diabetic patients. Res Nurs Health 1983:6:127-41.

19. Glasgow R, McCaul K, Shafer L. Barriers to regimen adherence among persons with type I diabetes. J Behav Med 1986;9:65-77.

20. Schlenk E, Hart L. Relationship between health locus of control, health value, social support, and compliance of persons with diabetes mellitus. Diabetes Care 1984;7:566-74.

21. Ludvigsson J, Richt B, Svensson P. Compliance and the health belief model: its relevance to the treatment of juvenile diabetes mellitus. Scand J Soc Med Suppl 1980;18:57-72.

22. Radius S, Becker M, Rosenstock I, Drachman R, Schuberth K, Teets $K$. Factors influencing mothers' compliance with a medication regimen for asthmatic children. J Asthma Res 1978;15:133-49.

23. Smith N, Ley P, Seale J, Shaw J. Health benefits, satisfaction and compliance. Patient Educ Couns 1987;10:279-86

24. Folger J. Relationship of children's compliance to mothers' health beliefs and behavior. J Clin Orthod 1988:22:424-26

25. Ginsburg H, Opper S. Piaget's theory of intellectual development. New Jersey: Prentice-Hall Inc, 1979:69-177.

26. Daneman D. When should your child take charge? Diabetes Forecast 1991:61-66.

27. Wysocki T, Meinhold PA, Abrams KC, et al. Parental and professional estimates of self-care independence of children and adolescents with IDDM. Diabetes Care 1992;15:43-51.

28. Gochman D. The measurement and development of dentally relevant motives. J Public Health Dent 1973;35:160-64 
29. Gochman D. Perceived vulnerability and its psychosocial context. Soc Sci Med 1977;11:115-20.

30. Harter S, Pike R. The pictorial scale of perceived competence and social acceptance for young children. Child Dev 1984;55:1969-82.

31. Garrison W, Biggs D. The diabetes pictorial scales: a direct measure of young children's knowledge, attitudes, and behavior relevant to their insulin-dependent diabetes mellitus. Diabetes Educ 1990;16:21-24.

32. Garison W, Biggs D. Young children's subjective reports about their diabetes mellitus: a validation of the diabetes pictorial scale. Diabetes Educ 1990;16:304-8.

33. Brown M, Haylor $M$. Nursing research with preoperational age children: the uses of standardized tests. J Pediatr Nurs 1989;4:19-25.

34. Herman B, Janz N, Becker M, et al. Determinants of seeking preconception care among women with diabetes. Diabetes 1991;40(suppl):418A.
35. Haynes R. Taylor D, Sackett D. Compliance in health care. Baltimore, Md: Johns Hopkins University Press, 1979.

36. Johnson S. Silverstein J, Rosenbloom A, Carter R, Cunningham W. Assessing daily management in childhood diabetes. Health Psychol 1986;5:545-64.

37. Reynolds L, Johnson S, Silverstein J. Assessing daily diabetes management by a 24-hour recall interview. The validity of children's reports. J Pediatr Psychol 1990;15:493-509.

38. Andrews F, Withey S. Social indicators of well-being: Americans' perceptions of life quality. New York: Plenum, 1976:370.

39. Nunnally J. Psychometric theory. New York: McGraw-Hill, 1978.

40. Revicki D. Quality of life and NIDDM. Diabetes Spectrum 1990;3:260-66. 